\title{
Development of Electric Vehicle Charging Infrastructure Based on Population
}

\section{S.Mani $^{1}$ | R.Raguraj ${ }^{1}$ | R.Harikaran ${ }^{1}$ | S.Hariramselvakanth ${ }^{1} \mid$ K.S.Gowthaman ${ }^{2}$}

${ }^{1}$ UG Scholar, Department of EEE, Government College of Engineering, Sengipatti, Thanjavur-613402, Tamilnadu, India. ${ }^{2}$ Assistant Professor, Department of EEE, Government College of Engineering, Sengipatti, Thanjavur-613402, Tamilnadu, India.

\section{To Cite this Article}

S.Mani, R.Raguraj, R.Harikaran and S.Hariramselvakanth and K.S.Gowthaman, "Development of Electric Vehicle Charging Infrastructure Based on Population", International Journal for Modern Trends in Science and Technology, Vol. 06, Issue 06, June 2020, pp.:14-16; https://doi.org/10.46501/IJMTST060604

Article Info

Received on 26-April-2020, Revised on 22-May-2020, Accepted on 25-May-2020, Published on 28-May-2020.

\section{ABSTRACT}

This research investigates electric vehicle(EV) charging behavior and aims to find the best method for its prediction in order to optimize the EV charging station(CS). This paper discusses several commonly used machine learning algorithm or $k$-Nearest Neighbor( $k-N N)$ to predict charging station based on population data records. According to the objective of the charging station planning, use the concept of group to do clustering evolution search. Hence the results of $k-N N$ algorithm achieved through MATLAB software. Based on the population, the initial time location of the charging station will be randomly considered in Manapparai, Lalgudi, Vaiyampatti, Thiruverumbur in Trichy based on population.

\section{KEYWORDS: Electric Vehicle(EV), Charging Station(CS), $k$-Nearest Neighbor(k-NN),} MATLAB.

Copyright (C) 2014-2020 International Journal for Modern Trends in Science and Technology DOI: https://doi.org/10.46501/IJMTST060604

\section{INTRODUCTION}

The Indian government has recently started taking many initiatives for development of sustainable and easily accessible charging stations. Inappropriate positioning of charging stations may affect smooth operation of the power grid so voltage instability, increased power loss, harmonic and lower reliability indices. In this project, $\mathrm{k}-\mathrm{NN}$ algorithm are utilized to find the better placement charging station in our area i.e. Manapparai, Lalgudi, Vaiyampatti, Thiruverumbur in Trichy. In $\mathrm{k}-\mathrm{NN}$ algorithm, the best result is chosen for the better placement of charging station based on population in those areas.

\section{K-NEAREST NEIGHBOR (K-NN)}

k-Nearest Neighbor(k-NN) was introduced by Onel Harrison. It is simple and easy to implement machine learning algorithm that can be used to clear regression and classification problems. It is a best method of clustering. Also a random search algorithm based on the concept of natural selection process which is used in optimization problems. In that paper we considered population based charging station. It is easy to implement and by adjusting the parameters the best result is achieved as compared to Genetic Algorithm(GA) and Particle Swarm Optimization(PSO) in some criteria's only. It becomes makes efficient to use 
this method.

\section{LITERATURE SURVEY}

In Adaptive genetic algorithm, it adjusts the probability of mutation dynamically based on of population fitness value and it shows population diversity to clear premature problems. In that algorithm is efficient to solve the problems of premature.[1]

This paper presents initial population of clustering based on Particle Swarm Optimizer(PSO). It is an clustering integrated index, which combines the space location and fitness value, Simulation results of this method is effective.[2]

Obtained from the other approaches of algorithm, it has purity. Its simulation results of the proposed algorithm capable of yielding optimized solution with better quality and high purity degree in comparison of all other algorithms.[3]

This paper focuses on the feature selection of Particle Swarm Optimization(PSO). Its problems are designed by initialization of filter based population methods. k-Nearest Neighbor(k-NN) are used for assessment performance of feature selection.

In results of proposed method shows the improvement performance of Particle Swarm Optimization(PSO) of problem selection of features.[4]

Size of population may be critical in applications of Genetic Algorithm(GA) and Particle Swarm Optimizer(PSO). It have the probabilities of mutation and crossover on the population of diversity and convergence of algorithm. It makes great process of searching in global optimization. [5]

Variation of traditional PSO algorithm, it provides the Efficient Population Strategy for PSO (EPUS-PSO), population to manage the adaptation improving the significantly efficiency of PSO.[6]

Its results indicate the new algorithm advantages of better convergence and give the implementation of the steps of algorithmin multi-population composite genetic algorithm and improved self-adaptive algorithm.[7]

\section{ISSUES IN THE EXISTING}

Genetic Algorithm(GA) and particle swarm optimization (PSO) are cyclically repeated process. In Genetic Algorithm(GA) not find optimal solution problems in all cases. It becomes hard for choosing parameters like population size and number of generations etc.,

In PSO, it has found in the following steps are 1. Initialize the group of particles, 2. Evaluate pbest for each particle, 3.To find current position is better than pbest or not, it becomes true update the positions otherwise to find pbest to gbest, 4.to compute the velocity, 5.update the particle position, it becomes true the target is reached otherwise go to step 2 . In this algorithm cyclically repeated and consuming more time.

\section{PROPOSED METHODOLOGY}

The proposed system should be helpful to find the optimal solution of charging station based on population. It is the best method for clustering process compared to other algorithm. k-NN algorithm find the best place of selecting charging station with minimum cost. The results of this algorithm is efficient to all and utilize all people for optimized areas. In this algorithm, assumption on this hinges close to be true enough. Our childhood days we uses to find the distance from points on a graph and simple method.

\section{FLOW CHART}

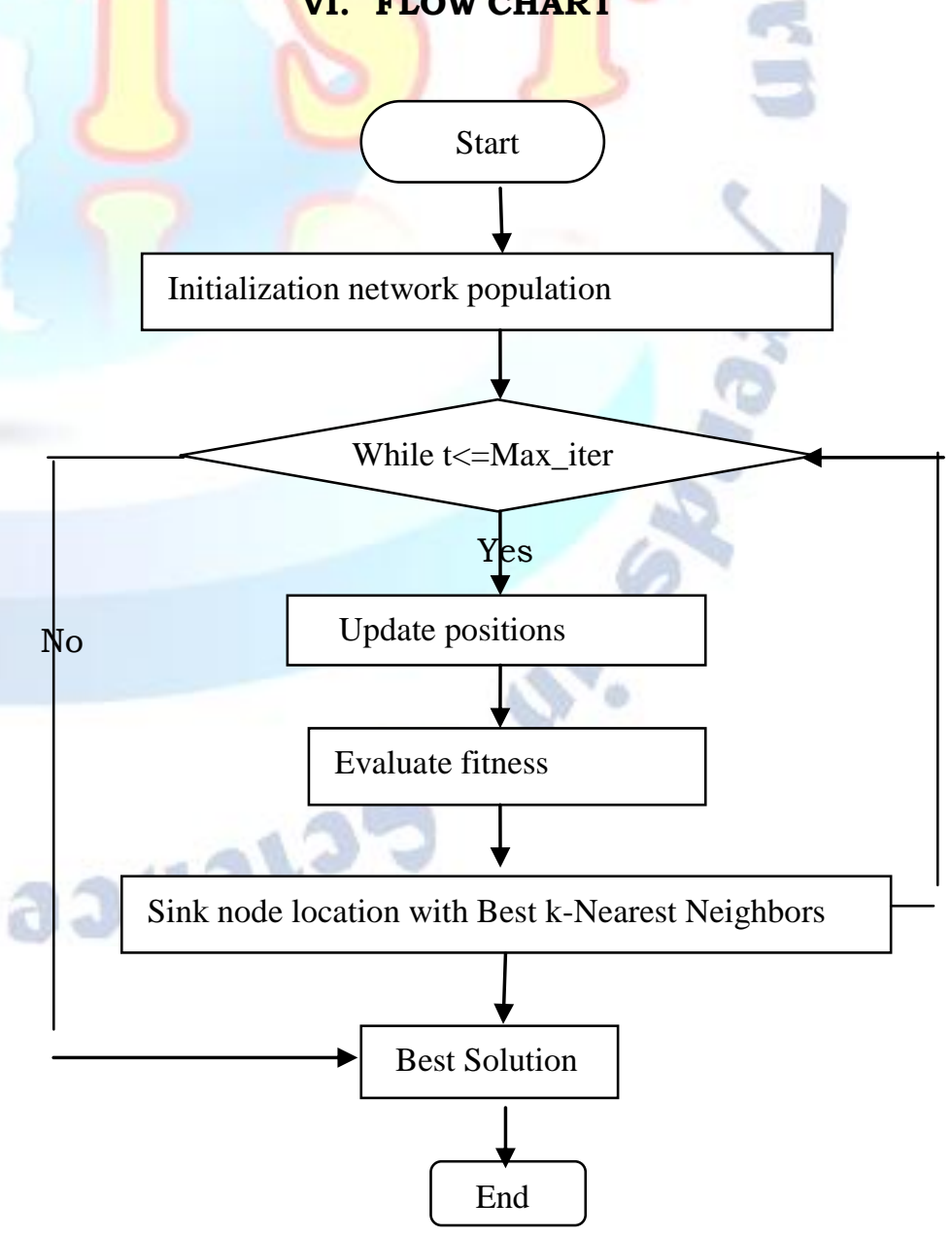

Fig:6.1 Flow chart 
In the above flow chart we clearly know about the $\mathrm{k}-\mathrm{NN}$ algorithm. it stores the dataset and make it the real time predictions. It captures the ideas of classification i.e. based on population, distance and closeness etc., it is easy to implement and it requires of $\mathrm{k}-\mathrm{NN}$ algorithm one is $\mathrm{k}$ value another one is total distance function of areas. It analyze the charging station in represented areas and it becomes very efficient.

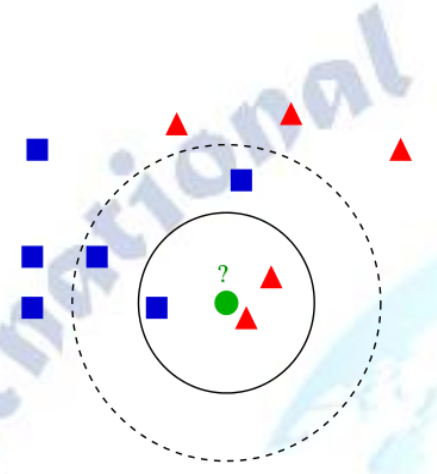

Fig: 6.2 Model k-NN algorithm

In model $\mathrm{k}-\mathrm{NN}$ algorithm blue square and red triangles found in there. If we give the value of $k=3$ it analyze the blue square and red triangles to find the best solution. based on $\mathrm{k}$ values the results were depend on there.

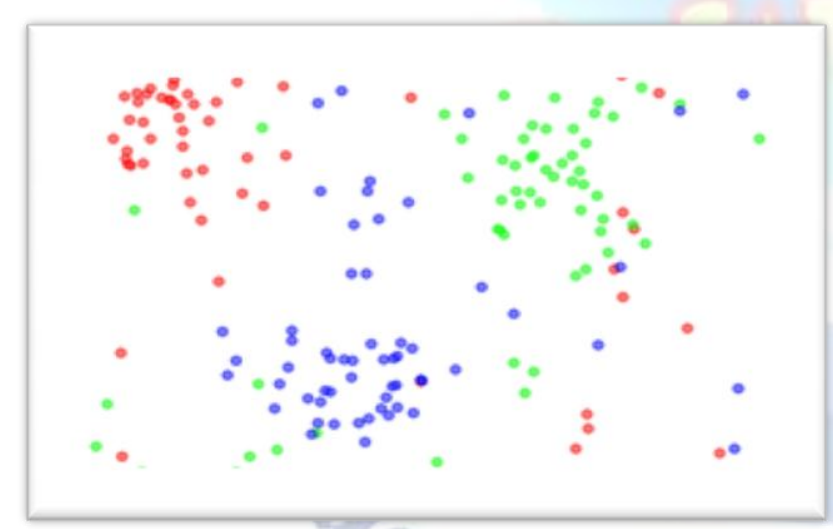

Fig:6.3 Dataset of k-NN algorithm

\section{CLUSTERING}

It is the samples of large data based on classification categories and express a best result in that areas, the input of data can be split in different sets. It improves the spectral clustering process and effective solution of approximation.
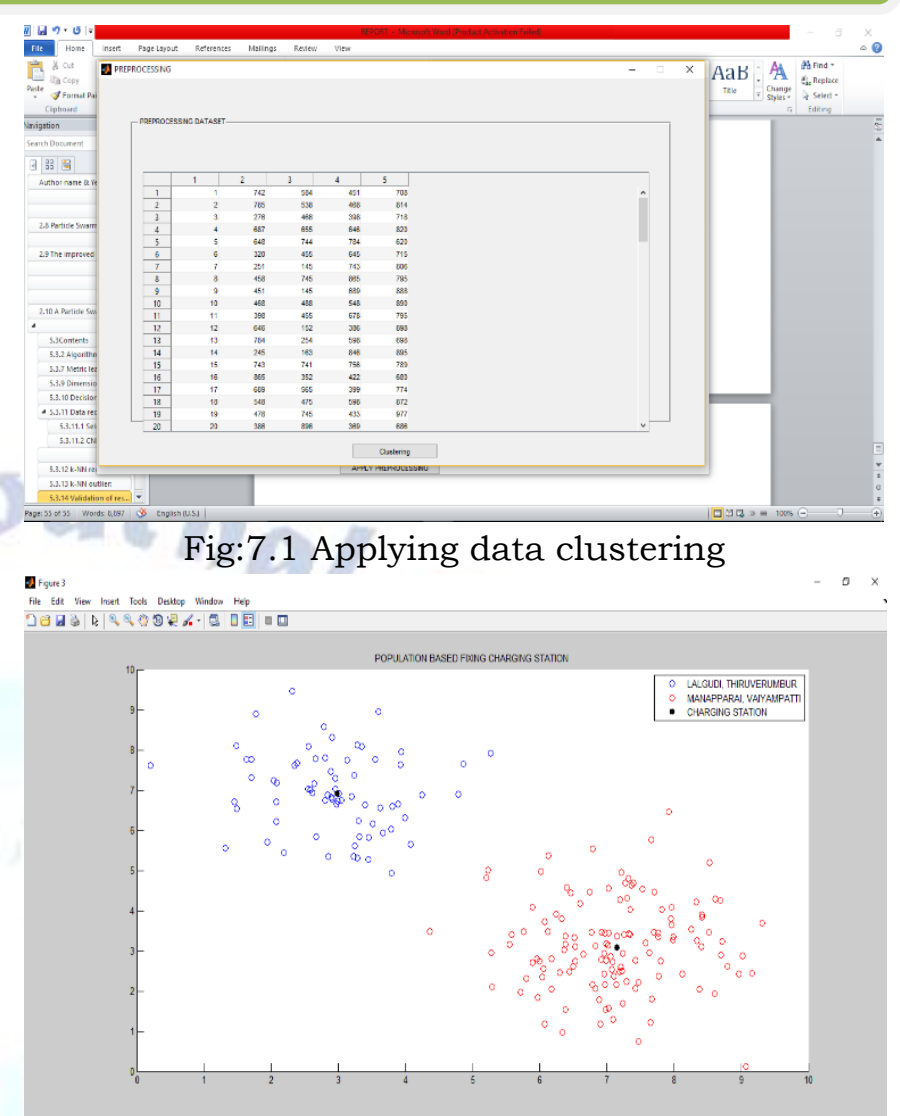

Fig:7.2 Fixing charging station

VIII. CONCLUSION

Vehicles are a major contributor to pollution in cities and their replacement with EVs will definitely improve air quality. In this project, we make alternative method of transportation i.e.EVs. our program is based on the population for choosing charging station at appropriate location.

\section{REFERENCES}

[1] Shweta Taneja, Charu Gupta, Kratika Goyal, Dharna Gureja.,[2014]. " An Enhanced k-Nearest Neighbor algorithm using information gain and clustering”. International Conference on Advanced Computing and Communication Technologies.

[2] Shichao Zhang, Xuelong Li, Ming Zong, Xiaofeng Zhu, Ruli Wang.,[2017]. "Efficient kNN Classification with Different Numbers of Nearest Neighbor". IEEE Transactions on Neural Network and learning Systems.

[3] SanjeevikumarPadmanaban, Ramazan Bayindir, Eklas Hossain.,[2019]. "Electric Vehicle charging station location analysis and determination”. IEEE 9 september 2019.

[4] Huawei Yang, Yabiao Gao, Kathleen Blair Farley, Mike Jerue, Jason Perry, Zion Tse., [2015]. "EV usage \& city planning of charging station installations". 2015 IEEE Wireless Power Transmission Conference.

[5] Mark Kibanov, Martin Becker.,[2018]. "AdaptivekNN Using Expected Accuracy for Classification of Geo-Spatial Data". IEEE April 9-13,2018.

[6] Li-Yu Hu, Min-Wei Huang, Shih-Wen Ke and Chih-Fong Tsai.,[2016]. " The Distance Function effect on k-Nearest Neighbor classification for medical datasets.Springeplus 9 August 2016. 